\title{
Abstracts From the ISTS Satellite Meeting June 20, 2016, Brisbane
}

\section{DOES SEDENTARY BEHAVIOR INCREASE THE RISK OF LOW BACK PAIN? A POPULATION-BASED CO-TWIN STUDY OF SPANISH TWINS}

Anita B. Amorim ', Gavin M. Levy', Francisco Peréz-Riquelme², Milena Simic', Evangelos Pappas', Amabile B. Dario', Manuela L. Ferreira ${ }^{3}$, Eduvigis Carrillo ${ }^{4}$, Alejandro Luquez-Suarez ${ }^{4}$, Juan R. Ordonaña ${ }^{5}$, and Paulo H. Ferreira ${ }^{3}$

${ }^{I}$ Faculty of Health Sciences, The University of Sydney, Sydney, NSW, Australia ${ }^{2}$ Murcia Health Council, Murcia, Spain

${ }^{3}$ The George Institute for Global Health, Sydney Medical School, The University of Sydney, Sydney, NSW, Australia

${ }^{4}$ Murcia Twin Registry, Department of Human Anatomy and Psychobiology, University of Murcia, and IMIB-Arrixaca, Spain

${ }^{5}$ Discipline of Physiotherapy, University of Málaga, Málaga, Spain

Background: Low back pain (LBP) is the most prevalent musculoskeletal condition, contributing to an enormous financial burden worldwide. Only in Spain, the annual average cost in lost working days for LBP is over $\$ 180$ million. In Australia, back pain is the leading cause of work loss days, costing around $\$ 4.8$ billion each year for healthcare. Current interventions for LBP demonstrate low to moderate improvements for pain and function. Therefore, the identification of the factors that increase the risk of LBP, along with prevention strategies, is crucial. Modern living has increased the tendency of people to adopt a more sedentary lifestyle, negatively impacting an individual's health. In addition, to further contribute to the multidimensional nature of LBP, there is now increasing evidence that genetic factors play an important role in the development of this condition. Hence, it is important to consider whether sedentary behavior is associated with, or increases the risk of developing LBP, particularly when considering the role of genetics and shared environment. Purpose: Our aim was to investigate if sedentary behavior is associated with the lifetime prevalence of chronic LBP and the risk of developing chronic LBP, care-seeking due to LBP, and activity limiting LBP when genetics and early environmental factors are accounted for. Methods: This was a crosssectional and longitudinal study with a within-pair twin case-control design. Two thousand, one hundred and forty-eight twins were included in a cross-sectional, and 1,098 in a longitudinal analysis. Sedentary behavior was the explanatory variable. Lifetime prevalence of LBP was the outcome variable in the cross-sectional analysis. The incidence of chronic LBP, care-seeking due to LBP, and activity limiting LBP were the outcome variables for the longitudinal analysis. Results: In the cross-sectional analysis, sedentary behavior was slightly associated with an increased prevalence of chronic LBP in females but not in males. This association was not apparent when genetics and early environmental factors are accounted for. In the longitudinal analysis, sedentary behavior did not significantly increase the risk of chronic LBP, nor care-seeking due to LBP, or activity limiting LBP. Conclusions: Sedentary behavior is associated with concurrent LBP. However, this association is weak, as it only appears in females and does not stand clearly when genetics is accounted for. Future studies using a twin design with larger samples should be conducted to replicate these findings.

\section{CURRENT CHALLENGES OF STUDIES 'OF' TWINS, 'BY' TWINS, AND ‘FOR' TWINS IN JAPAN}

\section{Juko Ando}

Keio University, Tokyo, Japan

A twin study consists of three different, but interrelated, research interests: studies 'of' twins, which investigate specificity of twin birth, twin relationship, and so on; studies 'by' twins, which provide a major methodology of behavior genetics to investigate how genes and environments work in human; and studies 'for' twins, which provide useful information for nurturing and fostering twin children in their families and societies. Main works in studies 'of' twins are on twin birth rate, zygosity diagnosis, and physical development. These findings also contribute to studies 'for' twins, which provide scientific references of social support for twin and their parents. Most of the active research region is study 'by' twins. There are two established twin research centers progressing: Osaka University Twin Research Center and Keio Twin Research Center. The former is medical oriented and focuses mainly on population from adolescent to elderly people. The latter is psychology oriented and focuses on population from infancy to adulthood. Currently, these two contrasted twin projects started to collaborate with each other to establish a nation-wide network for twin research in Japan.

\section{BULLYING AND VICTIMIZATION IN TWINS: THE EFFECT OF CLASSROOM SHARING AND OTHER TWIN-SPECIFIC FACTORS}

Meike Bartels, Sabine A. M. Veldkamp, Elsje van Bergen, Toos C. E. M. van Beijsterveldt, and Dorret I. Boomsma

Department of Biological Psychology, Vrije Universiteit, Amsterdam, the Netherlands

Peer bullying and victimization is a widespread phenomenon among school-age children and can have detrimental effects on the development of children. Relatively little is known about bullying and victimization in twins. This study examined whether twins are at high or low risk compared to their non-twin siblings and if several twin-specific factors, such as classroom sharing, zygosity, or gender composition, have an effect on bullying and victimization. This study included a large group of twins $(N=9,909)$ and their related non-twin siblings $(N=1,534)$ aged $7-12$ from the Netherlands Twin Register. Bullying and victimization was based on a four-item 
scale filled out by the teacher. Prevalence rates did not differ between twins and singletons. In total, $36 \%$ of children had been bullied moderately to severely and $35 \%$ suffered moderately to severely from victimization. Boys were more likely to bully and were at higher risk to become a victim than girls. Importantly, female twin pairs placed together in the same class did not bully more often, but were victimized less often $(O R=0.617)$. No effects of zygosity or gender composition of the twin pairs were observed. Previous studies found no harmful or beneficial effects of classroom sharing on traits such as school performance. In contrast, our results for victimization indicate that it might act protectively to assign female twins to the same class.

\section{GENOME-WIDE METHYLATION STUDY OF WELLBEING IN DISCORDANT MONOZYGOTIC TWINS}

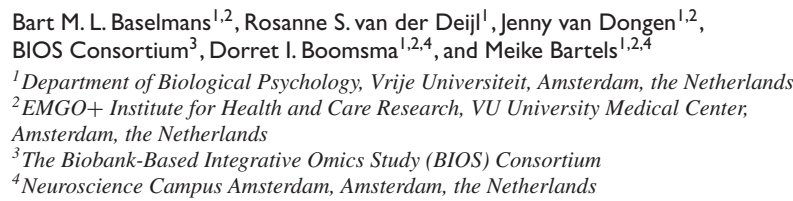

Wellbeing (WB) is a major topic across many scientific disciplines, mainly driven by its putative preventive role for mental and physical health. A recent meta-analysis of twin studies yielded an average weighted heritability of WB of $36 \%$ and $32 \%$ for satisfaction with life (SAT). These estimates show that WB has a substantial genetic component, but also show that environmental influences need to be investigated in order to explain individual differences in WB. The importance of the interplay between genes and environment has been well recognized in complex traits, yet most studies on WB have only investigated genetic and environmental components separately. Possible important moderators of gene-environment interactions are mechanisms of epigenetic gene regulation, of which DNA methylation has been most widely studied. This mechanism involves the modification of DNA through the binding of a methyl group to the cytosine of a $\mathrm{CpG}$ dinucleotide. Depending on the genomic region which is methylated, the presence of a methyl group is associated with the modification of gene expression. Recently, the first epigenome-wide association study (EWAS) for WB has been performed, which found two $\mathrm{CpG}$ sites for which methylation levels were genome-wide significantly associated with WB after Bonferroni correction. To further investigate the methylation differences associated with WB, we conducted an EWAS in $14 \mathrm{MZ}$ twins discordant for SAT score. Participants were selected from the Netherlands Twin Register (NTR). For each subject, we selected SAT scores that were measured closest in time to the moment of blood draw. We considered twins as discordant for WB when this score was 16 or lower (equivalent to a 5 on the Quality of Life scale) and the cotwin score was 28 or higher (equivalent to a 7 on the Quality of Life scale). DNA methylation was measured with the Infinium HumanMethylation450 BeadChip. To test for differences in methylation within twin pairs, we performed paired $t$-tests on each probe. The outcome variables for these tests were the residual methylation levels, which were calculated from the initial $\beta$ levels by adjusting them for many covariates, including different blood cell counts, age, and smoking. While none of our probes showed genome-wide significance after Bonferroni correction, $\Delta \beta$ and $p$ values were in the range of similar study designs. Thirty-eight sites had a $p$ value $<1 \times 10^{4}$, and three sites had a $p$ value $<1 \times 10^{5}$. In these probes, high $\Delta \beta$ were found in discordant twin pairs, but not in concordant twins. Moreover, in a random set of $\mathrm{CpG}$ sites, we found no such differences in $\Delta \beta$ between discordant and concordant twins. Our study highlights the role epigenetic alterations in the etiology of wellbeing. It also underlines the strength of the discordant twin design for epigenome-wide association studies.

\section{HERITABILITY OF BORDERLINE PERSONALITY FEATURES}

\section{IN AN AUSTRALIAN SAMPLE}

Lucia Colodro Conde', lan Gizer ${ }^{2}$, Tim Trull $^{3}$, Michael Lynskey',

Nicholas Martin', and Sarah E. Medland

${ }^{I}$ QIMR Berghofer Medical Research Institute, Brisbane, QLD, Australia

${ }^{2}$ University of Missouri, Columbia, MO, USA

${ }^{3}$ King's College London, London, UK

Aims: Borderline personality disorder (BPD) is a severe mental disorder characterized by a pervasive dysregulation of emotion as well as behavior, including suicidal behaviors, instability of relations, and self-image and impulse control. Its core feature is affective instability (AI). This disorder is associated with extreme emotional, behavioral, and interpersonal dysfunction, and its heritability estimates vary between $30 \%$ and $70 \%$. BPD features and AI are present at different degrees in non-clinical populations. This study aimed to disentangle the sources of variance of both traits in an Australian sample. Methods: The sample was comprised of 5,720 individuals including 612 MZF, 456 DZF, 307 MZM, 208 DZM, and 417 DOS complete pairs, with ages ranging from 18 to $80(M=34$, $S D=11)$ recruited as part of studies of health and wellbeing by QIMR. They completed the Personality Assessment Inventory Borderline Features Scale (PAI-BOR) of 24 items. We computed the square root transformation of the average score of the PAI-BOR total score and the AI subscale of the questionnaires. We modeled the sources of variance of both traits using structural equation modeling in OpenMx. Age, age $^{2}$, sex, and their interactions were used as covariates. Results: The twin correlations pointed to an ADE model, where the phenotypes are explained by additive (A) and dominant (D) genetic effects and unique environmental factors (E), including measurement error. The best fitting model showed that $47 \%$ (CI 95\% $=43-52 \%$ ) of differences observed in BPD features and $35 \%$ (CI 95\% = 30-40\%) in AI were due to A. The remaining variance was explained by $\mathrm{E}$ in both cases. Conclusions: Using an Australian sample, both BPD features and AI were shown to be moderately heritable. Our results are consistent with those found by Distel et al. (2009), who found that BPD features were due to additive $(21 \%)$ and dominant $(24 \%)$ genetic factors in an extended twin-family design with Dutch and Belgian samples. These results support the search of genetic variants associated with these traits.

\section{RELATIONSHIP BETWEEN FAT TASTE THRESHOLDS AND BODY MASS INDEX IN TWINS}

Andrew Costanzo', Caryl Nowson ${ }^{2}$, Liliana Orellana ${ }^{3}$, and Russell S. Keast ${ }^{I}$ Centre of Advanced Sensory Science, Deakin University, Melbourne, VIC, Australia ${ }^{2}$ Institute of Physical Activity and Nutrition, Deakin University, Melbourne, VIC, Australia

${ }^{3}$ Faculty of Health, Deakin University, Melbourne, VIC, Australia

The sense of taste influences, via quality and hedonics, the intake of energy and nutrients. Recent evidence indicates that there is a taste quality responsive to fat that is assessed by measuring fatty acid thresholds in the oral cavity. Fat taste thresholds (FTT) have been correlated with energy intake and dietary fat content, with high thresholds associated with high dietary fat. The relationship between FTT and body mass index (BMI) is more controversial with only some studies showing an association. Aims: To identify the withinpair association of FTT between monozygotic (MZ) and dizygotic (DZ) twin pairs, and to identify the association between FTT and BMI. Methods: MZ and DZ twin pairs (18-65 years) were recruited via the Australian Twin Registry to participate in this study to assess FTT and BMI. FTT for oleic acid (C18:1) were determined using an ascending concentration 3-Alternate Forced Choice methodology. This method involves subjects tasting a series of ascending concentrations of milk samples containing C18:1. Milk 
samples are presented in sets of three: one contains C18:1 and two controls. FTT is the concentration at which subjects are able to correctly identify the taste of C18:1 three times in a row. Height was measured using a stadiometer and weight was measured using electronic scales. Results: Forty-two pairs of twins (33 MZ, 9 DZ), 65 female and 19 males (mean age 42.4 [15.6] [SD] years; mean BMI $26.3[5.5] \mathrm{kg} / \mathrm{m}^{2}$ ), were assessed for FTT (C18:1). The mean FTT was $6.72(8.22) \mathrm{mM}$. There was a significant correlation for FTT within MZ twin pairs $(n=33, r=0.388, p=.034)$, whereas within DZ pairs $(\mathrm{n}=9)$ there was no significant correlation $(r=0.216, p=.577)$ (Spearman's correlations). Overall, the correlation between FTT and BMI was not significant $(n=84, r$ $=-0.020, p=.863)$, within MZ twin pairs the correlation was $r$ $=0.131(p=.491)$ and within DZ twin pairs was $r=0.167(p=$ .667). The within-pair BMI correlation in MZ twin pairs was $r=$ $0.896(p<.001)$ and in DZ twin pairs was $r=0.500(p=.170)$. The within-pair height correlation in MZ twin pairs was $r=0.965$ $(p<.001)$ and within DZ twin pairs was $r=0.420(p=.260)$. Conclusion: The within-pair correlation for FTT suggests that there is likely to be a genetic contribution to FTT, indicating that the ability to detect the level of fat within a food is influenced to some extent by genetic factors. However, a greater number of DZ pairs are required to confirm this conclusion. There was no relationship between FTT and BMI overall or by zygosity, indicating that ability to detect the level of fat within a food is not related to BMI during midlife.

\section{STUDIES OF THE ROLE OF EPIGENETICS IN THE EARLY LIFE ORIGINS OF DISEASE USING A TWIN COHORT}

Jeffrey M. Craig' , Richard Saffery', Yuk Jin Loke', Anna Czajko', Boris

Novakovic' ', Eric J. Joo' ', Alicia Oshlack' , Lavinia Gordon', John Galati' , Michael Permezel $^{2}$, Euan Wallace ${ }^{3}$, and Mark Umstad ${ }^{4}$

${ }^{I}$ Murdoch Childrens Research Institute, Royal Children's Hospital, Melbourne, VIC, Australia

${ }^{2}$ The Ritchie Centre, Monash Institute of Medical Research, Melbourne, VIC, Australia ${ }^{3}$ Department of Obstetrics and Gynaecology, University of Melbourne, Mercy Hospital for Women, Melbourne, VIC, Australia

${ }^{4}$ Department of Obstetrics and Gynaecology, University of Melbourne, Royal Women's Hospital, Melbourne, VIC, Australia

Background: The Peri/postnatal Epigenetic Twins Study (PETS) is a unique cohort of 250 mothers and their twins. Women were recruited from three Melbourne hospitals midway through their second trimester, which enabled measurement of maternal and fetal factors at multiple time points. We collected multiple biospecimens at birth (cord blood, cords, placenta, and buccal tissue) and repeat samples of blood and buccal swabs when infants were 18 months and 6 years of age. We aimed to study the plasticity of epigenetic marks and the genes they control during the intrauterine period and in early childhood. Methods: We performed both gene-specific and genome-wide analysis of the epigenetic mark of DNA methylation in multiple cell types using Sequenom MassArray EpiTyper and Illumina Infinium arrays. Data were regressed against multiple shared maternal factors (e.g., IVF, folate intake, gestational diabetes) and placenta weights, specific to each twin. Results: Twin pairs exhibited a wide range of within-pair epigenetic discordance at birth, which overlapped with that observed between unrelated individuals. Using gene-specific analysis, we found that certain gene ontologies are consistently variably methylated within pairs in all tissues, and that genetic and intrauterine environmental influence on DNA methylation varied throughout the genome. Using regression analysis, we identified genes whose expression and methylation levels correlated with birth weight in MZ pairs. These were enriched in functions related to nutrient metabolism and response to environmental agents. We also found an association between placental weight and gestational diabetes and DNA methylation at birth and tissue-specific association with other factors. Conclusions: Our data support the idea that genetic, shared (maternal) and non-shared (pla- cental) environmental factors impact on the developing epigenome. They also suggest that multiple early environments may be epigenetically reprogramming genes involved in metabolism, which may provide a mechanism for the early origins of cardiometabolic disease.

\section{CAN WE DO MORE TO HELP PARENTS OF NEWBORN TWINS UNDERSTAND ABOUT ZYGOSITY AND CHORIONICITY?}

Tessa L. Cutler' ${ }^{\text {, Jeffrey M. Craig }}{ }^{2,3}$, Nancy L. Segal ${ }^{4}$, Mark P. Umstad ${ }^{5}$, Louise A Keogh $^{6}$, Monica Rankin ${ }^{7}$, Jane Denton ${ }^{8}$, Catherine A. Derom ${ }^{9,10}$, Athula Sumathipala ${ }^{11,12}$, Jennifer R. Harris ${ }^{13}$, and John L. Hopper ${ }^{1,14}$

${ }^{1}$ Australian Twin Registry, Melbourne School of Population and Global Health, The University of Melbourne, Melbourne, VIC, Australia

${ }^{2}$ Murdoch Childrens Research Institute, Royal Children's Hospital, Melbourne, VIC, Australia

${ }^{3}$ Department of Paediatrics, University of Melbourne, Melbourne, VIC, Australia

${ }^{4}$ Department of Psychology, Twin Studies Center, California State University

Fullerton, CA, USA

${ }^{5}$ Royal Women's Hospital, Parkville, Australia; Department of Obstetrics and Gynaecology, The University of Melbourne, Melbourne, VIC, Australia

${ }^{6}$ Centre for Health Equity, Melbourne School of Population and Global Health, The University of Melbourne, Melbourne, VIC, Australia

${ }^{7}$ International Council of Multiple Birth Organisations (ICOMBO), Melbourne, VIC, Australia

${ }^{8}$ The Multiple Births Foundation, Queen Charlotte's \& Chelsea Hospital, Imperial College NHS Healthcare NHS Trust, London, United Kingdom

${ }^{9}$ Department of Human Genetics, University Hospital Gasthuisberg, KU Leuven, Leuven, Flanders, Belgium

${ }^{10}$ Belgium Association for Scientific Research in Multiple Births, Ghent, Belgium ${ }^{11}$ Research Institute for Primary Care and Health Sciences, Faculty of Health, Keele University Staffordshire, Keele, UK

${ }^{12}$ Sri Lankan Twin Registry, Institute for Research and Development, Colombo, Sri Lanka

${ }^{13}$ Division of Epidemiology, The Norwegian Institute of Public Health, Oslo, Norway ${ }^{14}$ Department of Epidemiology, School of Public Health, Seoul National University, Seoul, South Korea

Aims: For many years, twin research has provided insights into the genetic and environmental origins of health and disease. Such studies have relied on estimates of zygosity based on sex, chorionicity, and phenotypic similarities. However, the extent to which twins and parents of twins are informed about and understand zygosity is unknown. We aimed to ascertain the levels of zygosity knowledge held by adult twins and parents of twin children, to determine the basis of zygosity knowledge and to understand what zygosity knowledge means to twins and their families. Methods: At the 2012 Australian TwinsPlus Festival, we offered zygosity testing for twins who were unsure about their zygosity status. Buccal DNA was sent to the Australian Genome Research Facility for a 12-marker short tandem repeat zygosity test and results (monozygotic or dizygotic) were fed back to participants. Participants completed one short questionnaire at the time of providing the buccal sample and another after they received the zygosity results. These questionnaires were completed by adult twin participants and parents of child twin participants. Qualitative and quantitative information was collected about the basis of their zygosity knowledge (e.g., doctors, observations), as well as the importance and impact of the knowledge. Results: Of the 125 pairs from which a cheek swab was obtained, more than $90 \%$ were found by DNA testing to be monozygotic. One in five of the tested twins had incorrectly thought they were dizygotic. One in three said that they had received misinformation from medical professionals. Incorrect calls were based on the false assumptions that two placentas meant dizygotic twins and/or that monozygotic twins always look identical. Parents and adult twin pairs alike said that it was very important for them to know the true zygosity and many were surprised at the results. The reasons most frequently provided regarding the importance of zygosity knowledge were (1) for certainty; (2) for identity; and (3) for health reasons. Conclusions: There are misunderstandings about knowledge of zygosity by twins and parents of twins that appear in part to come from medical professionals. We recommend that (1) all parents of newborn same-sex 
twins in Australia be provided with written information on the twins' chorionicity and zygosity wherever possible; (2) that all same-sex twin pairs should have zygosity tests unless medical professionals provide written evidence that twins shared the same placenta; (3) twins and parents of twins should have easy access to information about how to be zygosity tested and what results mean; (4) medical professionals should receive further education about determining zygosity to reduce the misinterpretation on placenta arrangements; and (5) information about the cost of available zygosity tests is readily available in consideration of the greater financial costs association with raising twin children compared with singletons.

\section{THE AUSTRALIAN TWIN REGISTRY HEALTH AND LIFESTYLE QUESTIONNAIRE}

Tessa L. Cutler, John L. Hopper, Katrina Scurrah, and Lucas C. Ferreira Australian Twin Registry, Melbourne School of Population and Global Health, The University of Melbourne, Melbourne, VIC, Australia

Aims: In 2014, the Australian Twin Registry (ATR) began administering an online Health and Lifestyle Questionnaire (HLQ) to ATR members. The questionnaire contained demographic questions and covered a broad range of health conditions and risk factors. The questionnaire aimed to enable research and provide improvements to the ATR member experience by (1) allowing the ATR to better describe its membership; (2) enabling identification of potential participants for specific research studies to reduce costs to researchers and reduce unnecessary approaches to ineligible ATR members; and (3) fast-tracking research by providing de-identified data to researchers for ethically approved research analyses. Methods: The design of the HLQ incorporated advice from twin researchers and validated questionnaires were used where possible. Many of the demographic and health questions were selected to enable comparison with Australian Bureau of Statistics data. All parents of twin pairs aged 1 to 18 and twins aged 18 or above were eligible to participate in the HLQ and were invited to complete the questionnaire. The email invitation contained a link to the online questionnaire which would take approximately 20 minutes to complete. New members and existing members who provide an email or reach the eligible age will continue to be approached indefinitely. Results: Over 6,100 adult twins, including over 1,500 pairs and over 3,000 parents of twin children have completed the HLQ. The responses to the survey have provided information on the zygosity of each pair from three sources; selfreport, DNA test result, and validated zygosity questionnaire. The results include lifestyle factors and current medical conditions for each twin, which allowed analysis of the incidence as well as concordance within pairs. Examples of information obtained included self-reported health rating, diabetes, asthma, epilepsy, psychological wellbeing, mental illness, cancer, vision problems, smoking, and alcohol consumption. Conclusions: Responses to the HLQ have allowed identification of over 700 twin pairs with specific medical conditions to meet the eligibility requirements of four studies currently recruiting through the ATR. With this information, the ATR has been able to use targeted approach methods to invite participants, allowing more participants to be recruited in less time. Identification of appropriate twin pairs has also provided important information for funding applications, both in Australia and internationally. Data from the HLQ is available for use in twin health research either as de-identified data or to be linked with ATR or other datasets. Data from the HLQ has already been provided to researchers for three national projects and one international project either as unaccompanied de-identified data or as a linked dataset for analyses. The ATR has been collaborating with the recently established Brazilian Twin Registry (BTR) to implement a HLQ to BTR members.

\section{OBESITY DOES NOT INCREASE THE RISK OF CHRONIC LOW BACK PAIN: A PROSPECTIVE STUDY OF SPANISH ADULT TWINS}

Amabile B. Dario', Manuela L. Ferreira ${ }^{2}$, Kathryn Refshauge ${ }^{3}$, Alejandro Luque-Suarez ${ }^{3}$, Juan R. Ordoñana ${ }^{4}$, and Paulo H. Ferreira' ${ }^{I}$ Discipline of Physiotherapy, Faculty of Health Sciences, The University of Sydney, Sydney, NSW, Australia

${ }^{2}$ The George Institute for Global Health, Sydney Medical School, The University of Sydney, NSW, Australia

${ }^{3}$ Discipline of Physiotherapy, University of Málaga, Málaga, Spain

${ }^{4}$ Murcia Twin Registry, Department of Human Anatomy and Psychobiology, University of Murcia, Murcia, Spain

Aim: Obesity is commonly investigated as a potential risk factor for low back pain (LBP); however, the current evidence remains unclear. To better understand the effects of obesity on LBP, our aim was to investigate whether obesity-related measures increase the risk of chronic LBP outcomes using a longitudinal design. We assessed obesity through measures that considered the magnitude, as well as the distribution of body fat mass. A within-pair twin case-control analysis was used to control for the possible effects of genetic and early shared environmental factors on the obesity-LBP relationship. Method: Prospective observational study using data from the Murcia Twin Registry in Spain. Participants were 1,098 twins, aged 43 to 71 years, who did not report chronic LBP at baseline. Followup data on chronic LBP (>6 months), activity limiting LBP and care seeking for LBP were collected after 2 to 4 years. Risk factors investigated were body mass index (BMI), percentage of fat mass, waist circumference, and waist-hip ratio. Sequential analyses were performed using logistic regression controlling for familial confounding: (1) total sample analysis (twins analyzed as independent individuals); (2) within-pair twin case-control analyses (all complete twin pairs discordant for LBP at follow-up); withinpair twin case-control analyses separated for (3) dizygotic and (4) monozygotic twins. Results: No increase in the risk of chronic LBP was found for any of the obesity-related measures: BMI (OR 0.99; 95\% CI 0.86 to 1.14 ), \% fat mass (OR $0.87 ; 95 \%$ CI 0.66 to 1.14 ); waist circumference (OR $0.98 ; 95 \%$ CI 0.74 to 1.30 ); and waisthip ratio (OR $1.05 ; 95 \%$ CI 0.81 to 1.36 ). Similar results were found for activity limiting LBP and care seeking due to LBP. After the adjustment for genetics and early environmental factors shared by twins, the non-significant results remained unchanged. Conclusion: Obesity-related measures do not increase the risk of developing chronic LBP or care seeking for LBP, with or without adjustment for familial factors such as genetics in adults. Our findings do not support a causal direct relationship between obesity and chronic LBP.

\section{ESTIMATING THE INFLUENCE OF GENETIC FACTORS ON THE EXPERIENCE OF PRIMARY DYSMENORRHOEA IN ADOLESCENCE AND YOUNG ADULTHOOD USING A TWIN STUDY}

Theresa Donnelly', Yuxi Chen' ${ }^{\prime}$ Minh Bui ${ }^{2}$, Aneeka Bott ${ }^{\prime}$, Tiina Jaaniste ${ }^{1,3}$, John Eden ${ }^{3}$, and David Champion ${ }^{1,3}$

${ }^{I}$ Department of Pain and Palliative Care, Sydney Children's Hospital, Sydney, NSW, Australia

${ }^{2}$ School of Population Health, University of Melbourne, Melbourne, VIC, Australia ${ }^{3}$ School of Women's and Children's Health, University of New South Wales, Sydney, NSW, Australia

Aims: The aims of this investigation were to understand the contribution of genetic factors on the experience of primary dysmenorrhoea (PDM) in adolescence and young adulthood. PDM has been reported to affect up to $90 \%$ of adolescents (10-20\% reporting severe suffering; Berkley, Pain: Clinical Updates, Vol. 21, 2013, pp. $1-8)$. PDM refers to pain during menstruation in the absence of underlying pelvic pathology. Commonly, pain is accompanied by associated symptoms including headaches, nausea, and backache. 
Family history of menstrual pain is a risk factor for PDM, in addition to lifestyle factors (e.g., diet, nulliparity, and younger age; Ju et al., Epidemiologic Reviews, Vol. 36, 2014, pp. 104-113). Our aim was to investigate evidence for genetic factors to influence the recognized PDM components: pain, associated symptoms, and level of pain interference. Methods: We studied 226 female twins aged $11-22($ mean $=17, S D=2.97)$. The sample was drawn from a subset of twins who met our inclusion criteria: being female twin pairs aged 11 years and older from a larger cohort who had already responded to a mailed survey via the Australian Twin Registry. Monozygous (MZ) twins made up 57\% of the sample. Measures included an adapted version of the Retrospective Symptom Scale to assess lifetime prevalence of bothersome period pain and associated symptoms. Pain was also assessed on a current cycle using a numerical rating scale completed daily for each day up to 7 days on a single menstrual period. Pain interference with activities of daily living was also assessed using the PROMIS Pain Interference Scale. Heritability (h2) was estimated using the variance component model based on the classic twin model (using SOLAR software). Results: Adjusted for age, non-pain PDM symptoms was heritable (h2 $\pm S E=0.58 \pm 0.29$ ); as well as pain intensity, as indicated both by highest pain on a current menstrual period $(0.66 \pm$ $0.06)$ and by average pain $(0.63 \pm 0.07)$; and level of pain interference $(0.51 \pm 0.28)$. Heritability was not significant $(p=.076)$ for the lifetime measure of bothersome period pain. Conclusions: The results indicated that the experience of non-pain symptoms as part of the PDM symptom complex, as well as the intensity of period pain and the level of pain interference could be influenced by genetic variants. The experience of bothersome period pain recalled over the lifetime surprisingly was not estimated to be influenced by genetic variants; however, this may have been attributable to the use of a scale that restricted variability in possible ratings. These results overall are consistent with genetic influence contributing to the experience of PDM, notably including the experience of associated symptoms.

\section{GENETIC AND ENVIRONMENTAL INFLUENCES ON DIETARY INTAKE AND BODY WEIGHT: THE IMPORTANCE OF MONOZYGOTIC TWINS IN THE QUEBEC NEWBORN TWIN STUDY}

\section{Lise Dubois}

School of Epidemiology, Public Health and Preventive Medicine, University of Ottawa, Ontario, Canada

The objective of this study was to document the association between dietary intake at 9 years and subsequent Body Mass Index (BMI) in adolescent monozygotic boy and girl twin pairs. This research used data from 152 twin pairs. Dietary data were collected from two 24-hour-recall interviews with a parent and the child aged 9 years. Height and weight were obtained when the twins were aged $9,12,13$, and 14 years. Intrapair variability analysis was performed to identify dietary elements related to BMI changes in subsequent years. BMI-discordant monozygotic twin pairs were also identified to analyze the dietary constituents that may have generated the discordance. After eliminating potential confounding genetic factors, pre-adolescent boys who ate fewer grain products and fruit and consumed more high-fat meat and milk had higher BMIs during adolescence; pre-adolescent girls who consumed more grain products and high-fat meat and milk had higher BMIs during adolescence. Energy intake at 9 years was not related to BMI in subsequent years. In this presentation, we also explore next steps using genetic sequencing. The exonic and regulatory regions of 13 candidate genes in the IGF family had been sequenced (i.e., IGF1, IGF2, INS, and associated receptors (INSR, IGF1R, IGF2R) and binding proteins (IGFBP1 to IGFBP6); and SORCS2, a gene associated with circulating IGF1 and IGFBP3). One hypothesis for this project is that breastfeeding duration can mitigate the effect of IGF family variants on obesity development in late childhood and adolescence.

\section{PARENTAL REPORTS OF INFANT AND CHILD EATING BEHAVIORS ARE NOT AFFECTED BY THEIR BELIEFS ABOUT THEIR TWINS' ZYGOSITY}

Moritz Herle', Alison Fildes ', Fruhling Rijsdijk ${ }^{2}$, and Clare H. Llewellyn '

${ }^{I}$ University College London, London, UK

${ }^{2}$ Institute of Psychiatry, King's College London, London, UK

This is the first study to examine if parental reports of their infant and toddler twins' behavior were biased by their beliefs about their zygosity. Data were from Gemini, a British birth cohort of 2,402 twin pairs born in 2007. Twins' zygosity was assessed using the following: (1) a 20-item validated parent report questionnaire completed when twins were 8.0 months old $(S D=2.1)$, and again at 28.8 months $(S D=3.3)$; and (2) DNA. Parents were asked if they believed their twins were monozygotic (MZ) or dizygotic (DZ). 220/749 MZ pairs were misclassified by parents as DZ; 6/800 DZ pairs were misclassified as MZ. Intraclass correlations (ICCs) for a range of parent reported eating behaviors (four measured at 8 months, five at 16 months) were of the same magnitude for both correctly classified and misclassified MZ pairs. This suggests parents are not influenced by their twins' zygosity when reporting on the eating behaviors of infants and young children.

\section{THE INTERNATIONAL NETWORK OF TWIN REGISTRIES (INTR): INTEGRATED COLLABORATIVE EFFORTS FOR TWIN RESEARCH}

Chika Honda' and Lucas Ferreira ${ }^{2}$

${ }^{\prime}$ Osaka University, Osaka, Japan

${ }^{2}$ University of Melbourne, Melbourne, VIC, Australia

The International Network of Twin Registries (INTR) is a working group of the International Society for Twin Studies (ISTS) aimed at fostering scientific collaboration and promoting twin research globally through the expansion of existing resources of twin registries across the world (Buchwald et al., Twin Research and $\mathrm{Hu}$ man Genetics, Vol. 17, 2014, pp. 574-577). This session will have two presentations. Initially, it will report on the 4th INTR Meeting held in Osaka, Japan, and will discuss the main takeaways from this event. Second, Worldwide Twins, an online platform planned to fulfill some of the INTR objectives will be presented. Report of the 4th INTR Consortium Meeting at Osaka University, Japan. Representing 16 countries worldwide, more than 50 researchers gathered at Osaka University, Japan, in September 2015. Participants presented their recent research achievements, and the efforts and struggles in establishing and running registries were shared. International collaboration is also a vital element of INTR. A followup of successful research collaboration was presented, and several plans for future partnership among researchers were explored during the meeting. The summary of the meeting and the meaningfulness of the INTR consortium will be discussed. Worldwide Twins: an online platform to facilitate global twin studies. Worldwide Twins will be an online platform based on previous efforts within the INTR with the following objectives: to facilitate global twin studies; to support the creation of new twin registries across the world; and to disseminate information more broadly to diverse stakeholders (twins and their families, researchers, and society in general) who have interests in learning about and developing twin research. This presentation aims to discuss the rationale behind the platform, showcase a few examples of how it will operate and how twin research will benefit from it. It also intends to obtain valuable 
feedback from the twin research community and make a call for new collaborators.

\section{TWIN RESEARCH FOR POPULATION HEALTH AND WELLBEING}

\section{John L. Hopper}

University of Melbourne, Melbourne, VIC, Australia

The Australian Twin Registry is currently supported by a Centre for Research Excellence grant from the National Health and Medical Research Council (NHMRC), whose vision is 'to improve the health and well-being of all Australians'. In this talk, I will present ideas on how twin studies can be a unique and powerful tool for health and medical researchers to uncover not just causes of variation, but causes per se, both genetic and environmental. In this regard, addressing the absolute magnitudes of components of variations, and of risk gradients, could be more informative than constructs based on percentages, such as heritability. Now that it is possible to measure multiple new putative risk factors, in particular using new 'omics' technologies, within-pair twin designs are highly relevant to achieve the NHMRC vision. Twin cohorts provide exceptional opportunities, not just due to their unique matching, but also due to the ability to identify high-risk individuals for etiological research under the banner of 'precision medicine', including 'precision prevention and screening'. Examples relevant to breast cancer will be presented. I will also demonstrate how inference on causation can be made by trying to falsify the classic multifactorial twin model though examination of familial confounding (ICE FALCON). This method applies a regression-based analysis of twin data on measured risk factors and outcome, using the cotwin's predictor as a proxy instrumental variable. Application of this to large twin cohorts now available, especially through population linkage studies and global collaborations being generated through the International Network of Twin Registries, could see twin research help improve population health and well-being.

\section{FREQUENCY OF ATTENDANCE TO RELIGIOUS SERVICE MODERATES GENETIC AND ENVIRONMENTAL INFLUENCES ON PROSOCIAL BEHAVIOR}

Yoon-Mi Hur and Hoe-Uk Jeong

Department of Education, Mokpo National University, South Korea

The link between religiosity and prosocial behavior has been much disputed. The purpose of the current study was to investigate whether frequency of attendance of religious service moderates genetic and environmental influences on prosocial behavior in a sample of Nigerian adolescent twins (mean age $=14.5$ years, $S D=2.0$ years). One thousand, seven hundred and ninety-four dizygotic (DZ) and 624 monozygotic (MZ) twins completed the Prosocial Behavior (PB) scale of the Strengths and Difficulties Questionnaire and the question on frequency of attendance to religious service. Twins' zygosity was determined using 18 DNA markers. Univariate modelfitting analysis revealed that additive genetic and non-shared environmental influences accounted for individual difference in prosocial behavior. However, frequency of attendance to religious service was largely explained by shared environment and non-shared environmental influences. The phenotypic correlation between prosocial behavior and frequency of attendance to religious service was positive but modest $(r=0.13)$. The bivariate genotype by environment interaction model-fitting analysis revealed that relative influence of additive genetic factors in prosocial behavior increased with increasing frequencies of attendance to religious service. Taken together, our findings suggest that frequency of attendance to religious service is an environmental moderator that increases genetic effects on prosocial behavior.

\section{SWEET TASTE PERCEPTION IS ASSOCIATED TO BODY} MASS INDEX AT THE PHENOTYPIC AND GENOTYPIC LEVEL

Liang-Dar Hwang ${ }^{1,2}$, Gabriel Cuellar-Partida ${ }^{1,2}$, Jue-Sheng Ong ${ }^{1,2}$,

Paul A. S. Breslin ${ }^{3,4}$, Danielle R. Reed ${ }^{3}$, Stuart MacGregor' , Nicholas G. Martin', and Miguel E. Renteria'

${ }^{I}$ QIMR Berghofer Medical Research Institute, Brisbane, QLD, Australia

${ }^{2}$ School of Medicine, University of Queensland, Brisbane, QLD, Australia

${ }^{3}$ Monell Chemical Senses Center, Philadelphia, PA, USA

${ }^{4}$ Department of Nutritional Sciences, School of Environmental and Biological Sciences, Rutgers University, New Brunswick, NJ, USA

Studies investigating the relationship between body mass index (BMI) and sweet taste perception have been inconclusive. We conducted a longitudinal study using a genetic informative sample to determine any relationship between BMI and sweet taste. First, we estimated the phenotypic correlation between perception of four different sweet compounds (glucose, fructose, neohesperidine dihydrochalcone (NHDC), aspartame) and BMI in a sample of 1,576 adolescent Australian twins. Second, we computed the association between adolescent taste perception and BMI in early adulthood. Finally, we performed twin modeling and used a polygenic risk prediction approach to investigate the presence of any genetic overlap between BMI and sweet taste. We observed limited evidence of association between taste perception and BMI measured at the same time (adolescence), with the strongest evidence of association observed for glucose with a correlation coefficient of $r=0.06$ ( $p=$ $.029)$ and for aspartame with $r=0.06(p=.035)$. BMI measured 8 years later was significantly associated with each of the taste perception scores, with the strongest sweet compound correlation observed in aspartame with $r=0.09(p=.007)$. Finally, we found a significant genetic correlation between perception of glucose and NHDC and BMI. Our analyses show that adolescent sweet taste perception can be a potential indicator of BMI in early adulthood. This association is further supported by evidence of genetic overlap between the traits, suggesting that the action of some genes on BMI may act through their effects on taste perception.

\section{GENETIC AND ENVIRONMENTAL ASSOCIATIONS BETWEEN SELF-EFFICACY AND DEPRESSIVE SYMPTOMS IN A JAPANESE POPULATION}

Fujio Inui ${ }^{1}$, Karri Silventoinen ${ }^{1,2}$, Chika Honda $^{3}$, Rie Tomizawa ${ }^{3}$, Kae Nakatani ${ }^{4}$, and Kenji Kato ${ }^{5}$

${ }^{I}$ Faculty of Health Science, Kio University, Osaka University, Osaka, Japan

${ }^{2}$ Department of Social Research, University of Helsinki, Osaka University, Osaka, Japan

${ }^{3}$ Osaka University Graduate School of Medicine, Osaka University, Osaka, Japan ${ }^{4}$ Faculty of Health science, Kio University, Nara, Japan

${ }^{5}$ Kobe City College of Nursing, Hyogo, Japan

Aims: In order to clarify the associations between self-efficacy and depressive symptoms, the present study examined the role of shared genetic and environmental factors underlying these traits. Methods: Study participants were derived from the Osaka University Twin Registry. Self-efficacy scores were measured by the General SelfEfficacy Scale (GSES) and depressive symptoms by the Center for Epidemiologic Studies Depression Scale (CES-D) and Geriatric Depression Scale short version (GDS). All raw scale scores were standardized for age and sex by calculating regression residuals. Results: The questionnaires were sent to 1,394 twin individuals (697 twin pairs), and a total of 720 individuals responded. Because of missing data, 53 individuals were excluded ( $20 \%$ or more missing data in each scale). All analyses were conducted with a sample of 667 twin individuals including 230 twin pairs (194 monozygotic and 36 dizygotic pairs) having the mean age of 59.9 years and a standard deviation of 18.7 years. The data were analyzed using Cholesky decomposition by OpenMx statistical software. Both additive genetic and unique environmental factors contributed to the association between self-efficacy and depressive symptoms. The genetic 
correlation between GSES and CES-D was -0.44 (95\% CI -0.69 , -0.18 ) and between GESE and GDS -0.64 (95\% CI -0.81, -0.46). Unique environmental correlation between GSES and CES-D was -0.21 (95\% CI $-0.34,-0.08)$ and between GESE and GDS -0.32 (95\% CI -0.43, -0.19). Estimated path coefficients were as follows: additive genetic factor $\mathrm{A} 1$ to GSES $=16.0(\mathrm{CI}=10.7,21.7)$, CES-D $=-7.1(\mathrm{CI}=-12.1,-2.3)$ and GDS $=-5.5(\mathrm{CI}=-7.9,-3.4), \mathrm{A} 2$ to $\mathrm{CES}-\mathrm{D}=16.1(\mathrm{CI}=7.8,23.3)$ and $\mathrm{GDS}=5.9(\mathrm{CI}=3.1,8.8)$, and $\mathrm{A} 3$ to $\mathrm{GDS}=4.7(\mathrm{CI}=3.2,6.3)$. Unique environmental factor $\mathrm{E} 1$ to $\mathrm{GSES}=23.7(\mathrm{CI}=19.7,28.9), \mathrm{CES}-\mathrm{D}=-6.2(\mathrm{CI}=-10.7,-$ 2.1) and GDS $=-4.0(\mathrm{CI}=-5.9,-2.3)$, E2 to CES-D $=35.7(\mathrm{CI}$ $=29.1,44.5)$ and GDS $=7.3(\mathrm{CI}=5.1,10.0)$, and $\mathrm{E} 3$ to GDS $=6.6(\mathrm{CI}=5.4,8.0)$. When three genetic models were compared (Cholesky decomposition, independent pathway model, and common pathway model), the common pathway model showed the best fit measured by Akaike information criterion. Conclusions: The results show that genetic and unique environmental factors contribute to the association between self-efficacy and depressive symptoms. Furthermore, a common pathway model expecting a latent factor behind self-efficacy and depressive symptoms explain the associations more clearly. The results may help to investigate the causes of depression.

\section{ASSESSING AND OPTIMIZING VARIANT CALLER FOR DNA SEQUENCING DATA USING TWIN INFORMATION \\ Jina $\mathrm{Kim}^{\prime}$ and Joohon Sung ${ }^{2}$ \\ ${ }^{I}$ Interdisciplinary program in Bioinformatics, Seoul National University, Seoul, South Korea \\ ${ }^{2}$ Complex Disease and Genome Epidemiology Branch, Department of Epidemiology, School of Public Health, Seoul National University, Seoul, South Korea}

Next-generation sequencing technology is one of the platform technologies that drive advances in human genetics. As the current DNA sequencing data process heavily relies on analytical algorithms and pipelines, we need to have proper methods of evaluating the accuracy of analytic tools. It is, however, still difficult to evaluate which is more accurate in determining variants for whole genome sequencing data. In this study, our objective was to assess several variant callers by pairwise comparison using monozygotic twin genomes and to find optimized error-filtering methods. We used whole genome sequencing data (Illumina, x60 each) of two twin pairs from the Healthy Twin Study, the prospective cohort study that has been collecting participants in South Korea. By comparing whole genome sequencing data from one monozygotic twin pair that has the identical genomes, we regard shared SNVs as true variants and discordant SNVs as errors. The following variant callers were used: GATK-HC(Genome Analysis Tool Kit Haplotype Caller),Platypus, and Samtools. Additionally, we explored which variant caller (e.g., initial screening for errors and confirming the variants) is optimal and effective using several statistics. Finally, we apply selected variant calling methods to one sample from the Coriell CEPH/UTAH 1463 family (FASTQ data), and to decide variants (vcf format). By comparing this final vef with the high-confident variant call set of NA12878 that was released as a reference genome material by the Genome in a Bottle (GIAB) consortium, we measured true performance of our optimized variant calling method. Our findings suggest that the GATK-HC has the best performance according to discordance rate (GATK-HC, $5.67 \%$; Platypus, $6.51 \%$ ), and the variant calling process method might be recommended for general use. This strategy of selecting higher performance tools might be able to apply to broader purposes too. We believe it is noteworthy to mention the main advantage of twin data over duplicated individual samples. The discordant variants mainly consist of errors, but small proportion of somatic mutations is included and the discordant loci will provide big research potential.

\section{GENETICS AND ENVIRONMENTS ON JOB-RELATED} STRESS

Hakyung Kim and Joohon Sung

Department of Epidemiology, School of Public Health, Seoul National University, Seoul, South Korea

Background and Purpose: Job-related stress is known as a risk factor of cardiovascular disease, musculoskeletal disease, and major depression disorder. However, to our knowledge, the role of genetic influences on job stress has not been investigated. This study aimed to estimate the heritability of job-related stress traits in the Healthy Twin Study, Korea (HTS), applying the abbreviated version of the Job Content Questionnaire (JCQ) developed by Karasek. Methods: From the HTS $(n=3,499)$, a twin-family cohort study, we selected 1,890 individuals who have employment status; housewives $(n=$ 886), students (146), and others with unemployed or unclear job status (587) were excluded. The job-related stress level measured by the JCQ consisted of decision latitude (nine questions), psychological job demand (five questions), and a question about the physical demand. In the 'demand-control model', job-related stress is explained by two dimensions: decision latitude and psychological job demand. The heritability is estimated for each domain of JCQ. AE, ACE, ADE, ACDE models were fitted after adjusting age and sex to select the best model that explains each trait. Variance component approaches, implemented in the SOLAR were used for analysis. Results: Study participants $(n=1,872$, mean age $=41.55$, men $53.63 \%$ MZ 278 pairs, siblings 508 pairs) was comprised of 481 non-manual and 975 manual workers, and 416 supervisory positions. JCQ domain scores of each participant were different by age group, sex, job categories, and level of education $(p<.05)$. Level of education was grouped by four categories: below 6 years, 7-12 years, university undergraduates, and over graduate-school. Differences of JCQ domain score in job categories were significant only for decision latitude. For decision latitude (DL), ACE was the best fitted model $(\mathrm{C}=0.11)$ where only shared environments were meaningful. For psychological job demand (PJD), ADE was the best explanatory model, and only non-additive genetic effects were significant $(\mathrm{D}=$ 0.38). Covariates such as demographics and socioeconomic factors explained 0.15 and 0.06 of all trait variances of DL and PJD. Conclusions: Our findings suggest that conventional genetic effects might not play major roles on job-related stress domains, if any. The role of non-additive genetic effects on PJD might need further analysis, particularly with personality traits that are also known to have nonadditive genetic influences.

\section{COTWIN-CONTROL AND MENDELIAN RANDOMIZATION APPROACHES: COMPARING TWO CAUSAL EFFECT MODELS USING OBESITY AND BONE HEALTH STUDY}

Soo Ji Lee' and Joohon Sung ${ }^{1,2}$

${ }^{1}$ Department of Genome Epidemiology, School of Public Health, Seoul National University, Seoul, South Korea

${ }^{2}$ Institute of Health and Environment, Seoul National University, Seoul, South Korea

Aims: The Mendelian randomization (MR) approach, replacing environmental risk factors with genetic instrumental variables, is widely accepted as a causal effect model. The cotwin-control method, particularly for monozygotic twins (MZ), has long been suggested as a powerful approach by matching genomic constitutions and nuisance environmental covariates, otherwise hard to adjust. In this study, we would like to compare the two approaches by empirically examining results from the two analyses using the Healthy Twin Study (HTS) in Korea. We chose general obesity, central obesity, and bone health as an example because conflicting reports are still submitted on this important public health issue, particularly according to menopause status. Methods: MR 
Analysis: To obtain valid IVs for different adiposity measures, we conducted genome-wide association (GWA) screens using two cohort studies in Korea (total 17,935). The MR analysis was performed using the HTS $(n=3,479)$. Body mass index (BMI) and waist-hip ratio adjusted for BMI (WHRaBMI) as a central obesity indicator were used as adiposity traits; and bone mineral content (BMC) and bone mineral density (BMD) measured by DEXA were used as bone health parameters. Cotwin-Control Analysis: We calculated the difference in BMC and BMD within MZ pairs and regressed those differences on the differences in BMI and WHRaBMI for the same pairs. The presence of meaningful associations and the estimated effect sizes were compared. Results: MR Analysis: Traitspecific GRSs were successfully identified for BMI and WHRaBMI ( $F$ statistics $>10$ ). Both GRSs showed randomized effects over the possible confounders. For weight-bearing bones, BMI IVs showed significant positive associations with BMC (g) (both spinal - men 10.41 [SE 4.19]; premenopausal women (PRE) 6.55 [SE 2.35], and lower limbs - men 23.31[SE 10.39]; PRE 20.75 [SE 6.29]) and BMD (lower limbs only - men 0.019 [SE 0.008]; PRE 0.016 [SE $0.005]$ ); these patterns were not evident for postmenopausal women. Central obesity IVs did not show significant associations with any of the bone parameters across all subgroups. Pooled analyses for all weight-bearing bones for both men and women showed positive BMI associations with BMC/BMD (20.7/0.013). Cotwin-Control Analysis: BMI showed significant associations with all bone parameters BMC/BMD (the estimated effects of 7.35 [SE 1.85]/0.004 [SE .001]); and no significant trend between cotwin difference of WHRaBMI and bone traits. Conclusions: Our findings suggest that both MR and cotwin-control analyses will be able to show causal associations by controlling confounders by randomization (MR) or matching (cotwin-control). Our findings also suggest that the estimated effect size might be smaller in cotwin-control analysis, probably because of the overmatching of shared environmental factors within twin pairs.

\section{A MEASURE OF GENOME-WIDE AVERAGE DNA METHYLATION, WHICH IS ALSO A RISK FACTOR FOR BREAST CANCER, IS DETERMINED IN UTERO: INSIGHTS FROM SEVEN TWIN AND FAMILY STUDIES OF DIFFERENT STAGES OF THE LIFESPAN}

Shuai $\mathrm{Li}^{1}$, Ee Ming Wong ${ }^{2}$, Pierre-Antoine Dugué ${ }^{3}$, Eunae $\mathrm{Kim}^{4}$, Ji-Hoon Eric Joo ${ }^{2}$, Allan F. McRae ${ }^{5}$, Roger L. Milne ${ }^{3}$, Carmel Apicella', Nicola J. Armstrong 6 , Perminder S. Sachdev ${ }^{7}$, Karen A. Mather ${ }^{7}$, Anbupalam Thalamuthu ${ }^{7}$, Margaret J. Wright ${ }^{8}$, David Ames ${ }^{9,10}$, Naomi Wray ${ }^{5}$, Grant W. Montgomery ${ }^{8}$, Timothy D. Spector ${ }^{11}$, Joohon Sung ${ }^{4,12}$, Jeffrey M. Craig ${ }^{13,14}$, Richard Saffery ${ }^{13,14}$, Jenny van Dongen ${ }^{15}$, Graham G. Giles ${ }^{1,4}$, Melissa C. Southey ${ }^{2}$, and John L. Hopper'

${ }^{I}$ Centre for Epidemiology and Biostatistics, Melbourne School of Population and Global Health, University of Melbourne, Melbourne, VIC, Australia

${ }^{2}$ Genetic Epidemiology Laboratory, Department of Pathology, University of Melbourne, Melbourne, VIC, Australia

${ }^{3}$ Cancer Epidemiology Centre, Cancer Council Victoria, Melbourne, VIC, Australia ${ }^{4}$ Department of Epidemiology, School of Public Health, Seoul National University, Seoul, South Korea

${ }^{5}$ Centre for Neurogenetics and Statistical Genomics, Queensland Brain Institute, University of Queensland, Brisbane, QLD, Australia

${ }^{6}$ Mathematics and Statistics, Murdoch University, Perth, Australia

${ }^{7}$ Centre for Healthy Brain Ageing (CHeBA), School of Psychiatry, University of New South Wales, Sydney, NSW, Australia

${ }^{8}$ QIMR Berghofer Medical Research Institute, Brisbane, QLD, Australia

${ }^{9}$ Academic Unit for Psychiatry of Old Age, Department of Psychiatry, University of Melbourne, Melbourne, VIC, Australia

${ }^{10}$ National Ageing Research Institute, Melbourne, VIC, Australia

${ }^{11}$ Department of Twin Research and Genetic Epidemiology, Kings College London, London, UK

${ }^{12}$ Institute of Health and Environment, Seoul National University, Seoul, South Korea

${ }^{13}$ Murdoch Childrens Research Institute, Royal Children's Hospital, Melbourne, VIC

Australia
${ }^{14}$ Department of Paediatrics, University of Melbourne, Melbourne, VIC, Australia

${ }^{15}$ Department of Paediatrics, University of Melbourne, Melbourne, VIC, Australia
${ }^{15}$ Department of Biological Psychology, VU Amsterdam, Amsterdam, the Netherlands

Aims: To estimate the genetic and environmental contributions to variation in a measure of genome-wide average DNA methylation across the lifespan. This measure has been shown by two prospective cohort studies to predict breast cancer risk, as well as do the currently known SNPs and mammographic density measures. Methods: We conducted seven twin and family studies in Australia, Korea, and the United Kingdom, comprising 2,518 individuals aged from 0 to 90 years. A measure of genome-wide average DNA methylation was derived from the Infinium HumanMethylation450 BeadChip assay, defined as the average beta-value across all probes. For this measure, we estimated familial correlations, modeled correlations as a function of cohabitation history, and fitted variance component models using a multivariate normal model fitted using the software FISHER. The estimations were performed within each study, and using all data ( $z$ score within each study) combined, respectively. Replication is planned using $\sim 2,500$ individuals from the Netherlands Twin Registry biobank project. Results: The genome-wide average DNA methylation measure was familially correlated for each study. The twin pair correlation was $\sim 0.8$, both at birth and age 18 months (both $p<10-30$ ), as high as the repeatability of this measure. The correlation decreased with age across adolescence $(p=10-6)$, and was constant at $\sim 0.4$ across adult life (all $p<10-4$ ). There was no evidence that the correlation differed according to zygosity across the lifespan $(p=.2)$. Using path coefficient analysis, the correlation between birth and the old age for an individual was predicted to be $\sim 0.7$. The correlation for (non-twin) first-degree relatives increased with time living together $(p=10-8)$, and tended to decrease with time living apart $(p=.09)$. The spouse pair correlation increased with time living together $(p<.02)$, and plateaued at $\sim 0.2(p=.02)$. From variance component modeling, there was no evidence for an additive genetic component from any study. When the data were combined, the estimate of the additive genetic component was $0 \%$ (95\% CI: $0-8, p=.3$ ); the environmental factors shared by twins alone explained 24\% (95\% CI: 16-32, $p=10-9$ ), and the environmental factors shared by all family members explained $17 \%(95 \%$ CI: $11-23, p=10-8)$ of variation in this measure. Conclusions: This measure of genome-wide average DNA methylation, which is enriched for gene-associated CpG sites and therefore potentially highly relevant to gene function and is a familial risk factor for breast cancer, is primarily determined by the time of birth. This is likely due to the shared environmental factors in utero, the effects of which persist into later life. It is also influenced by environmental factors shared by cohabiting family members, the effects of which attenuate when family members live apart. Genetic factors explain, at most, a small proportion of variation in this familial risk factor for breast cancer.

\section{TWIN AND GENOME-WIDE ASSOCIATION ANALYSIS OF NICOTINE METABOLITE RATIO, A BIOMARKER OF SMOKING BEHAVIOR}

Nicholas G. Martin', Gu Zhu', Anu Loukola ${ }^{2}$, Andrew Heath ${ }^{3}$ Pamela A. Madden ${ }^{3}$, Rachel F. Tyndale ${ }^{4,5}$, and Jaakko Kaprio ${ }^{6-8}$

${ }^{1}$ QIMR Berghofer Medical Research Institute, Brisbane, Queensland, Australia ${ }^{2}$ Department of Public Health, University of Helsinki, Helsinki, Finland ${ }^{3}$ Washington University School of Medicine in St. Louis, Missouri, USA ${ }^{4}$ Campbell Family Mental Health Research Institute (CAMH), Toronto, Ontario, Canada

${ }_{5}^{5}$ Departments of Pharmacology Toxicology and Psychiatry, University of Toronto, Ontario, Canada

${ }^{6}$ Department of Public Health, University of Helsinki, Helsinki, Finland ${ }^{7}$ Institute for Molecular Medicine (FIMM), University of Helsinki, Helsinki, Finland ${ }^{8}$ National Institute for Health and Welfare, Helsinki, Finland

Individuals with fast nicotine metabolism typically smoke more and thus have a greater risk for smoking-induced diseases. Further, the efficacy of smoking cessation pharmacotherapy is dependent on the rate of nicotine metabolism. Our objective was to use nicotine metabolite ratio (NMR), an established biomarker of nicotine metabolism rate, in a genome-wide association study (GWAS) to identify novel genetic variants influencing nicotine metabolism. 
A heritability estimate of 0.40 (95\% CI $0.09-0.63)$ was obtained for NMR using 19 complete pairs of MZ and 66 complete pairs of DZ twins from the Australian Twin Registry. We performed a GWAS in 1,551 cotinine verified current smokers among our adult twins and their relatives previously genome-wide, genotyped, and imputed using the 1000G HRC (reference set $>32,000$ Europeans). The strongest evidence for association emerged for CYP2A6 on $19 \mathrm{q} 13$ ( $\min p=8.58 \mathrm{E}-69$, in intron 4$)$, the main metabolic enzyme for nicotine. The results exactly replicate recent similar analyses in Finnish twins and Canadian samples, and we are currently conducting a meta-analysis to see if further significant loci can be identified, as well as to document the effects of these loci on smoking behaviors and smoking-related diseases.

\section{ASSOCIATION OF PSYCHIATRIC PROFILE MEASURES WITH BINOCULAR RIVALRY RATE (BRR): IMPLICATIONS FOR SLOW BRR AS AN ENDOPHENOTYPE FOR BIPOLAR DISORDER}

Trung T. Ngo', Baptiste Couvy-Duchesne ${ }^{1,2}$, Gabriel Cuellar-Partida ${ }^{1,3}$, Miguel E. Rentería', Lucia Colodro Conde', Scott D. Gordon'

Narelle K. Hansell' ${ }^{\text {, Phillip C. F. Law }}{ }^{3}$, Steven M. Miller ${ }^{3}$, Sarah E. Medland', Margaret J. Wright ${ }^{2}$, and Nicholas G. Martin'

${ }^{I}$ Genetic Epidemiology Laboratory, QIMR Berghofer Medical Research Institute, Brisbane, QLD, Australia

${ }^{2}$ Queensland Brain Institute, The University of Queensland, Brisbane, QLD, Australia ${ }^{3}$ The University of Queensland Diamantina Institute, Translational Research Institute, Brisbane, QLD, Australia

${ }^{7}$ Queensland Brain Institute, The University of Queensland, Brisbane, QLD, Australia 8 Monash Alfred Psychiatry Research Centre, Monash University Central Clinical School and The Alfred, Melbourne, VIC, Australia

${ }^{9}$ Monash Alfred Psychiatry Research Centre, Monash University Central Clinical School and The Alfred, Melbourne, VIC, Australia

Background: Binocular rivalry is a visual phenomenon in which simultaneous presentation of different stimuli, one to each eye, results in perceptual alternations or rivalry between the two images. Slow binocular rivalry rate (BRR) has been proposed as a potential endophenotype for the heritable psychiatric condition, bipolar I disorder (BD), because BRR is slower in BD than controls and is under substantial genetic influence $\left(\mathrm{h}^{2}=0.52\right)$ (Ngo et al., Acta Neuropsychiatr, Vol. 23, 2011, pp. 37-42). The BRR trait also shows high sensitivity $(\sim 80 \%)$ and test-retest reliability $(r$ $=0.70$ ), but its relation to medication and state requires clarification, as does its specificity given recent reports of anomalous BRR in depression, anxiety disorders, and attention deficit hyperactivity disorder (ADHD). Aim: To examine in a large twin sample, the relationship between subjects' BRR and their psychiatric profile measures. Methods: BRR was recorded and analyzed as per Miller et al. (PNAS, Vol. 107, 2010, pp. 2664-8) in 14-yearold twins $(N=1,143)$ participating in the Brisbane Longitudinal Twin Study (Wright \& Martin, Aust J Psychol, Vol. 56, 2004, pp. 65-78). In subsamples with BRR, there were also the following data: (1) the SPHERE (Somatic and Psychological HEalth REport), a 34-item self-rated screening tool for anxiety-depression and chronic fatigue $(N=954$; aged $14-14.6)$; (2) the SWAN (Strengths and Weaknesses of ADHD symptoms and Normal behavior) $(N=867)$, a DSM-V criteria-based 18 -item rating scale for inattention, hyperactivity, and impulsivity symptoms completed by mothers of the twins; and (3) diagnoses of lifetime major depressive disorder (MDD; $N=439,14 \%$ cases) and social anxiety disorder (SAD; $N=439,17 \%$ cases) based on the Composite International Diagnostic Interview (aged 18-29, mean=23). $R e$ sults: No association was found between subjects' BRR and their (1) anxiety-depression ratings $\left(r=-0.07, p=.11 ; \mathrm{R}_{\mathrm{G}}=-0.20\right.$, $p=.11)$, (2) ADHD symptoms $\left(r=-0.07, p=.09 ; \mathrm{R}_{\mathrm{G}}=-0.18, p\right.$ $=.10)$, (3) MDD diagnosis $\left(\mathrm{OR}_{\mathrm{MDD}}=1.20 ; p=.84\right)$, and (4) $\mathrm{SAD}$ diagnosis $\left(\mathrm{OR}_{\mathrm{SAD}}=0.23 ; p=.09\right)$. Conclusions: The findings lend support to the BRR trait as a potential endophenotype for BD by suggesting high specificity to BD and no relationship to anxiety- depression, however further work is required on these endophenotype issues with much larger datasets. We will also present preliminary results on the genetic correlation between BRR and psychiatric disorders (based on LD score regression analyses with Psychiatric Genomics Consortium datasets), and plans for large-scale studies of the trait using an online binocular rivalry testing platform (Law et al., Twin Res Hum Genet, Vol. 16, 2013, pp. 1033-1078).

\section{FINDING TWINNING GENES}

Dale R. Nyholt on behalf of the Twinning GWAS Consortium (TGC) Institute of Health and Biomedical Innovation (IHBI), Queensland University of Technology (QUT), Brisbane, QLD, Australia

Spontaneous dizygotic (DZ) twinning occurs in $1-4 \%$ of women, with familial clustering and unknown physiological pathways and genetic origin. DZ twinning indexes increased fertility and has distinct health implications for mother and child. Candidate gene association and genome-wide linkage studies have failed to uncover common variants associated with DZ twinning. Here, we report results from a genome-wide association (GWA) study in 1,980 mothers of spontaneous DZ twins and 12,953 controls. The GWA identified three SNPs significantly associated with DZ twinning $(p<$ $\left.5 \times 10^{-8}\right)$ of which two replicated $\left(p=3 \times 10^{-3}\right.$ and $\left.p=1.4 \times 10^{-4}\right)$ in a large independent Icelandic cohort. Subsequent analysis of the implicated DZ twinning risk alleles and a polygenic risk score for DZ twinning found a number of associations across a broad range of fertility traits in women, including age at menarche, age at first child, having children, age at last child, age at menopause, polycystic ovary syndrome, and serum FSH levels (Mbarek et al., American Journal of Human Genetics, Vol. 98, 2016, pp. 898-908). The implication of these results with respect to reproductive capacity and health will be discussed.

\section{IDENTITY FUSION IN TWINS}

Juan R. Ordonana', Encarna Tornero-Valero', Alexandra Vazquez-Botana ${ }^{2}$, Juan F. Sánchez-Romera', and Angel Gómez-Jiménez ${ }^{2}$

${ }^{1}$ University of Murcia, Murcia, Spain

${ }^{2}$ UNED, Madrid, Spain

Aims: Identity fusion is a visceral feeling of oneness with a group that predicts personally costly, pro-group behaviors (Swann et al., Psychological Review, Vol. 119, 2012, pp. 441-456). Fusion differs from identification and strongly fused persons maintain a sense of personal agency while aligning themselves with the group. This feeling of agency is strongly associated with pro-group actions, particularly when people perceive that group members share essential qualities as genes. For obvious reasons of genetic relatedness, twins are of special interest for the study of identity fusion processes. Also, hypothetical differences in fusion between twin zygosity could be relevant in the development of traits influenced by social support. Our aim was to analyze fusion and its relationship with psychological distress in a sample of adult middle-aged twins. Methods: The sample comprises 1,443 (503 MZ, 504 same-sex DZ, and 436 opposite-sex DZ) twins (mean age $=56.1, S D=6.8$ ). Fusion with the twin was assessed adapting a three-item (e.g., 'I am one with my twin') verbal scale (Response range: 0-10). Several measures of psychological distress were used: The depression/anxiety domain of the EQ-5D questionnaire; self-report of depression during last year, and use of anxiolytics or antidepressants. Results: MZ twins were significantly more fused with the twin than DZ twins (MMZ: 7.81, $S D: 2.1$; MDZ: $6.68, S D: 2.3 ; p<.001)$. Opposite-sex twins were not different in fusion with the twin that same-sex DZ twins (MDZOS: $6.59 ; S D: 2.3)$. Intra-class correlations for fusion were higher for MZ than for DZ twins (rMZ: 0.505, CI 95\% 0.401, 0.609; rDZ: 0.308, CI $95 \% 0.214,0.402$ ). None of the measures of psychological distress showed significant associations $(p>.05)$ with fusion, nor did other lifestyle (e.g., smoking or alcohol use) or wellbeing (i.e., 
health-related quality of life or subjective happiness) variables. Conclusion: As expected, fusion with the twin was significantly greater for MZ than for DZ twins, and the former showed greater resemblance than the latter. That has some implications for evolutionary and social or personality approaches to human behavior. Nevertheless, this feeling has no apparent impact on measures of psychological distress or wellbeing, which is consistent with the equal environments assumption.

\section{PROTECTIVE AND HARMFUL EFFECTS OF PHYSICAL ACTIVITY FOR LOW BACK PAIN: THE AUSTRALIAN TWIN BACK PAIN (AUTBACK) PILOT STUDY}

Marina B. Pinheiro', Manuela L. Ferreira ${ }^{2}$, Kathryn Refshauge', John Hopper ${ }^{3}$, Christopher Maher ${ }^{2}$, Jan Hartvigsen ${ }^{4}$, Bart Koes ${ }^{5}$, Markus Hübscher ${ }^{6}$, and Paulo H. Ferreira'

${ }^{1}$ Faculty of Health Sciences, University of Sydney, Sydney, NSW, Australia ${ }^{2}$ The George Institute for Global Health and Institute of Bone and Joint Research, The Kolling Institute, Sydney Medical School, University of Sydney, Sydney, NSW, Australia ${ }^{3}$ Centre for Epidemiology \& Biostatistics, Melbourne School of Population and Global Health, University of Melbourne, Melbourne, VIC, Australia

${ }^{4}$ Nordic Institute of Chiropractic and Clinical Biomechanics, Odense, Denmark ${ }^{5}$ Department of General Practice, Erasmus MC, University Medical Centre, Rotterdam, the Netherlands

${ }^{6}$ Neuroscience Research Australia and University of New South Wales, Sydney, NSW, Australia

Introduction: Although physical activity is a promising modifiable risk factor for low back pain (LBP), it is still not known what types and doses of physical activity are protective or harmful for LBP. Aims: To establish the feasibility of a definitive cohort study that will investigate the effects of different types and doses of physical activity on the risk of developing recurrent LBP while accounting for genetic factors. Specifically, we aim to determine the rate of recruitment, sample size requirements for the definitive study, the follow-up rate, compliance, completeness of data collection, and to identify possible barriers that could be experienced during the implementation of the study. Methods: This will be a longitudinal cotwin study. Twins will be recruited from the Australian Twin Registry and will be followed for 1 year. Fifty adult complete twin pairs with a history of LBP, but symptom-free at recruitment, will be included. Data on physical activity (predictor) will be collected using three questionnaires (Physical Workload Index, Recent Physical Activity, and International Physical Activity Questionnaire) and an objective measure (accelerometer - Actigraph) at baseline and at 6-month follow-up. Twins will also complete an additional physical activity questionnaire monthly (short form of the International Physical Activity Questionnaire). Data on LBP (outcome) will be collected at baseline and weekly. The main outcome will be the number of days that twins suffer from LBP. All data will be collected using short message service (SMS), email, and a specifically designed web-based platform. We will keep records of the recruitment rate, follow-up rate, and completeness of data. Barriers to completing the study will be investigated. Results: The Australian Twin Registry approached 655 complete twin pairs and 126 complete pairs expressed interest to be part of the study. Forty-eight complete pairs (38\%) met the study's inclusion criteria. The consent rate of twins meeting the inclusion criteria was $96 \%$. A total of 74 twins have completed all baseline measures, with monthly follow-up rates ranging from 1 to 4 months, and a follow-up completion rate of $87 \%$. All participants returned the Actigraph, with only two not meeting the recommended minimum wear-time. All questionnaires were created using a webbased tool and were extensively tested. Only one participant opted to receive a hard copy of the questionnaire. Overall, all study processes have been tested and no major issues were identified. Conclusions: The results of this study will inform the design and implementation of a future definitive study, which will help to clarify the effects of different types and doses of physical activity on the risk of developing recurrent LBP using an innovative design that controls for genetics and environment factors.

\section{A COMPARISON OF STILLBIRTH RATES IN MULTIPLE VERSUS SINGLETON PREGNANCIES, A MULTI-COUNTRY STUDY}

Monica Rankin

International Council of Multiple Birth Organisations (ICOMBO), Melbourne, VIC, Australia

Aim: While the incidence of stillbirths has decreased over the past decades, the rate is still much higher in twins and triplets than the rate for single births. The aim of this paper is to compare the incidence of stillbirths for single births and multiple births in a number of countries - Australia, Germany, United Kingdom, New Zealand, and the United States; and to ask researchers to investigate possible causes. It is hoped this will lead to improved outcomes for multiple birth pregnancies. Methods: Data were collected from various reputable sources on the annual number of births and stillbirths in Australia, Germany, United Kingdom, New Zealand, and the United States. Results: At the time of writing, data for the United States were unavailable. The following figures represent stillbirths in or around 2010. United Kingdom (Glinianaia et al., Twin Res Hm Genet, Vol. 16, pp. 112-116; NCCWH 2011: NICE Clinical Guidelines): Singletons, 5; Twins, 12; Triplets, 31. New Zealand (New Zealand Government Statistics): Singletons, 6; Twins, 21. Australia (Australian Bureau of Statistics): Singletons, 7; Twins, 19. Germany (Bundesamt, Wiebaden 2015): Singletons, 5; Twins, 9. It was difficult to obtain accurate data for any particular year for some countries. Triplet data were only available for the United Kingdom. Conclusion: The rate of stillbirth is significantly higher in multiples than in singletons. It may differ between countries, from two to three times higher in twins and perhaps as much as six times the rate in triplets. ICOMBO would like to see more data collected from a range of countries. Desirable outcomes for ICOMBO would include studies on why the stillbirth rate is significantly higher for multiples; how to improve the outcome for multiple birth pregnancies; the long-term impact of the death of part of a multiple pregnancy how does the family cope with the death of one twin while celebrating the birth of the co-twin and the longer term family relationships; and production of literature for families to assist them in coping with the stresses of stillbirth in a multiple pregnancy.

\section{THE OLDER AUSTRALIAN TWINS STUDY - FINDINGS FROM THE FIRST 10 YEARS}

Perminder S. Sachdev', Jocelyn L. Bowden', Teresa Lee', Wei Wen', David Ames $^{2}$, Bernhard T. Baune ${ }^{3}$, Henry Brodaty', John Crawford', Kristan Kang', Karen A. Mather ${ }^{1}$, Andrea Lammel ${ }^{1}$, Katherine Samaras ${ }^{4}$, Peter R. Schofield ${ }^{5}$, Anbupalam Thalamuthu', Julian N. Trollor ${ }^{6}$, and Margaret J. Wright ${ }^{7}$

${ }^{I}$ Centre for Healthy Brain Ageing, School of Psychiatry, University of New South Wales, Sydney, NSW, Australia

${ }^{2}$ National Ageing Research Institute, University of Melbourne, Melbourne, VIC, Australia

${ }^{3}$ School of Medicine, University of Adelaide, Adelaide, SA, Australia

${ }^{4}$ Garvan Institute of Medical Research, Sydney, NSW, Australia

${ }^{5}$ Neuroscience Research Australia, Sydney, NSW, Australia

${ }^{6}$ Department of Developmental Disability Neuropsychiatry, School of Psychiatry, University of New South Wales, Sydney, NSW, Australia

${ }^{7}$ Queensland Brain Institute, University of Queensland, Brisbane, QLD, Australia

Aims: To provide an overview of the findings from the Older Australian Twins Study (OATS) over the last 10 years. Methods: OATS is a major longitudinal study of twins, aged 65 years and over, to investigate genetic and environmental factors, and their interactions, in healthy brain aging and neurocognitive disorders. The study collects psychiatric, neuropsychological, cardiovascular, metabolic, biochemical, neuroimaging, genomic, and proteomic data, with 2 -yearly assessments. It is currently in its fourth data collection wave. The initial cohort comprises 623 individuals ( $158 \mathrm{MZ}$ and 124 DZ twin pairs; $1 \mathrm{MZ}$ triplets; 33 single twins and 23 non-twin siblings), of whom 450 have had a Wave 2 assessment, and 389 a Wave 3 assessment. An online extension of the study is proposed from 
Wave 4 onwards, with new recruitment planned. Results: A number of salient findings have emerged which assist in the understanding of genetic contributions to cognitive functions such as processing speed, executive ability, and episodic memory, and which support the cognitive reserve hypothesis. The heritability of brain structures, both cortical and subcortical, brain spectroscopic metabolites and markers of small vessel disease, such as lacunar infarction and white matter hyper-intensities, have been examined and can inform future genetic investigations. Work on amyloid imaging and functional magnetic resonance imaging is proceeding and epigenetic studies are progressing. Conclusion: This internationally important study has the potential to inform research in cognitive ageing into the future, and offers an excellent resource for collaborative work.

\section{TWINS SWITCHED AT BIRTH: A WORLD TOUR}

Nancy L. Segal

California State University, Fullerton, CA, USA

There have been seven documented cases of twins switched at birth. These rare cases offer unique opportunities to examine a number of key issues: genetic and environmental influences on the physical and behavioral development of reared-apart monozygotic (MZA) twins; phenotypic similarities and differences in unrelated siblings who believe they are dizygotic twins; emotional consequences of discovering the switch; the nature of the legal damages and compensation for switched twins and their families; the processes by which mothers identify their newborn babies; and hospital procedures developed to assure that mothers receive the correct newborns upon discharge. These areas will be explored with reference to past cases, as well as the most recently identified case, namely the doubly switched twin brothers of Bogotá, Colombia.

\section{GENETIC COVARIANCE BETWEEN THE CAROTID AND FEMORAL INTIMA-MEDIA THICKENING}

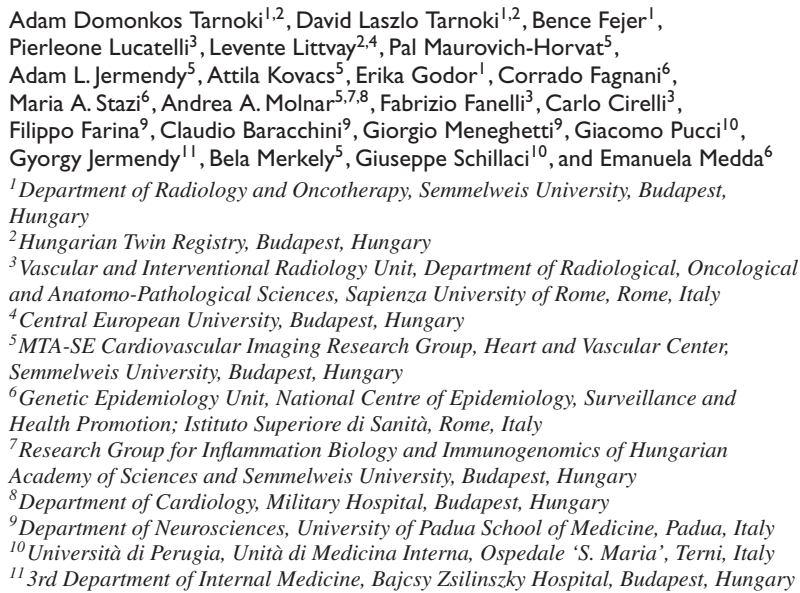

${ }^{5}$ MTA-SE Cardiovascular Imaging Research Group, Heart and Vascular Center, Semmelweis University, Budapest, Hungary

${ }^{6}$ Genetic Epidemiology Unit, National Centre of Epidemiology, Surveillance and Health Promotion; Istituto Superiore di Sanità, Rome, Italy

${ }^{7}$ Research Group for Inflammation Biology and Immunogenomics of Hungarian

Academy of Sciences and Semmelweis University, Budapest, Hungary

${ }^{8}$ Department of Cardiology, Military Hospital, Budapest, Hungary

${ }^{9}$ Department of Neurosciences, University of Padua School of Medicine, Padua, Italy

${ }^{10}$ Università di Perugia, Unità di Medicina Interna, Ospedale 'S. Maria', Terni, Italy

${ }^{11} 3 \mathrm{rd}$ Department of Internal Medicine, Bajcsy Zsilinszky Hospital, Budapest, Hungary

Aims: Increased carotid and femoral intima-media thickness (IMT) are prognostic factors of future cardiovascular events. We aimed to estimate the common genetic effects of carotid and femoral IMT in a relatively large twin sample. Methods: 388 Hungarian and Italian twins from Rome, Padua, Perugia, and Terni (121 monozygotic, 73 dizygotic pairs) underwent B-mode sonography of bilateral common carotid and femoral arteries (CCA, CFA). IMT was measured by semi-automated software (Philips QLAB), where available, or by calipers. Correlated factors model was applied. Results: Correlation between CCA-IMT and CFA-IMT was 0.26 (95\% CI $=0.112$ to 0.405$)$. Age-, sex-, and country-adjusted additive genetic factors for these parameters correlated well: 0.773 (95\% CI $=0.153$ to 1.0). Shared environmental correlation was found to be irrelevant. Unshared environmental factors did not correlate $(-0.021$,
95\% CI $=-0.239$ to 0.178$)$. Conclusions: Strong genetic correlation was found, indicating common genetic influences on CCA and CFA IMT. Further studies should be stimulated to find specific genes in this process. Atherosclerosis screening could be extended to various sites of vasculature due to this common genetic underpinning.

\section{HUNGARIAN TWIN REGISTRY: A 2016 UPDATE}

David L. Tarnoki ${ }^{1,2}$, Adam D. Tarnoki ${ }^{1,2}$, Levente Littvay ${ }^{1,3}$, Dora Melicher ${ }^{1,4}$, and Julia Metneki'

${ }^{1}$ Hungarian Twin Registry Foundation, Budapest, Hungary

${ }^{2}$ Department of Radiology and Oncotherapy, Semmelweis University, Budapest, Hungary

${ }^{3}$ Central European University, Budapest, Hungary

${ }^{4}$ Department of Genetics, Cell and Immunobiology, Semmelweis University, Budapest, Hungary

Aims: The first Hungarian twin registries established in the 1970s and 1980s ceased to exist in the 1990s. Efforts started in 2006 to revive and found a Hungarian twin registry (HTR) built on the previous databases, media appearances, and recruitments at twin meetings. Methods: To summarize the current activities of the HTR and the progress on efforts to expand it into a population-based registry. Results: Currently, the voluntary HTR consists of 1,000 adult twin pairs and multiples (50\% MZ, 50\% DZ, 70\% female). Efforts started in 2014 to expand the HTR into a population-based registry. In 2015, Levente Littvay was involved in CUPESSE study, an international family study of 10-12 countries investigating the entrepreneurial attitudes in 18- to 35-year-old subjects and their parents. As a twin sub-study, we received a fund from which we could start the build-up of the population-based HTR. Hungarian data authorities have been contacted, who provided the mailing address of 130,000 Hungarian citizens based on search with same mother's name, birth name, place, and date on a legal basis. The lecture will summarize the bureaucratic difficulties and the current update on the project. Conclusions: Based on the current status, the HTR will be expanded into a population-based twin registry, opening far-reaching possibilities to carry out ambitious scientific research in many areas. The realization is underway and it will be launched shortly, as soon as the bureaucratic issues are overcome.

PROVIDING FEEDBACK ON LONGITUDINAL PARTICIPATION AND RESULTS TO TWIN REGISTER PARTICIPANTS

Gonneke Willemsen', Hilde de Kluiver', Sara Khosdelazad', Jasper Bovenberg², and Dorret Boomsma'

${ }^{\prime}$ Department of Biological Psychology, Vrije Universiteit, Amsterdam, the Netherlands ${ }^{2}$ Director of the Legal Pathways Institute for Health and Bio Law, Aerdenhout, the Netherlands

As technology advances, it has become feasible to provide feedback to large groups of participants on the studies they participate in and their personal results. In addition, participants are increasingly aware of these possibilities and often actively pursue their right for return of results. In 2011, the Netherlands Twin Register (NTR) was one of the partners in BBMRI-NL, which worked on developing a participant portal and was the first to take the portal to its participants. Between March 2014 and 24 February 2016, log-in details for the MyNTR portal were sent by email in several batches to 18,476 participants, and 7,262 (39.3\%) of those activated their portal account. Participants responded positively to the portal, where they can view their own results (e.g., for sport participation, dental phobia, personality, pain) in relation to the average response of other participants. The portal also includes reports on overall study results (e.g., smoking, migraine, imaging studies) and details on the surveys participants completed. In addition, in the case of an open invitation for survey participation, the survey can be completed directly via the portal. In a pilot for a large non-response study, the reasons most stated for not activating the portal were having not seen the email 
or postponing activation till a more convenient moment. Examining participant characteristics showed that activation of the portal occurred more often for individuals who had completed more surveys, had participated recently, were female, and had higher educational attainment. Significant but small effects were also seen for personality: A higher score on conscientiousness, openness to experience, and agreeableness, and a lower score on neuroticism were related to more activation. The pattern of activation was similar for monozygotic and dizygotic twin pairs and for spouses, suggesting that portal activation is predominantly environmentally driven. In conclusion, a participant portal provides one way of encouraging participant activation and ensuring participants' rights are met. Future studies will need to examine the impact of portal activation on participation and survey responses. 